\title{
Optimization model of transport traffic at preliminary registration of queries based on controlled queueing models
}

\author{
Elizaveta Kondrashova ${ }^{1, *}$ \\ ${ }^{1}$ Moscow State University of Civil Engineering, Yaroslavskoe h. 26, 129327, Moscow, Russia
}

\begin{abstract}
The paper is devoted to the model of transport traffic optimization with use of preliminary registration. Preliminary registration of applications for entrance to a certain road-zone in certain time intervals can optimize a road situation and reduce traffic jams. The optimization is based on the controlled queueing model theory, controlled arrival-flow. As a result the transport flow becomes "predictable" and "controlled" thanks to preliminary information, using Internet-services. Algorithmization of model and also the main structure is presented. The example of control on the basis of controlled queueing model is given.
\end{abstract}

\section{Introduction}

Recently the transport flow optimization problems become more relevant. The problem of transport traffic: multi-hour traffic jams, overload of roads, increase of road traffic accidents, all it leads to the fact of traffic's becoming more difficult. It leads to a noneconomy of personal and work time of vehicular traffic participants.

Optimization at the expense of construction or expansion of roads in certain city areas in large cities such as Moscow, St.-Petersburg and others becomes technically impossible.

Thus, it becomes interesting formulation of the optimization problem of traffic (cars, buses, trucks et al.) while maintaining roads without additional construction and extensions, by managing traffic flow and setting criteria for the participants of the movement.

The object of management (control) in the system is logical to consider traffic flow, consisting of technical devices as cars, public transport, facilities executing freight traffic, etc. $[1,2,3]$.

\section{The major factors influencing traffic control}

Note, during traffic control one must, first, focus on some inalienable characteristics of vehicular traffic and second, that one needs to specify some optimization criteria, focusing on which it is possible to formulate and solve the optimal control problem.

During the city transport flow control usually the following characteristics are taken into consideration: the unsteadiness of transport flows, the stochasticity, the incomplete

*Corresponding author: elizavetakondr@gmail.com 
handling, the interrelatedness of multiple factors simultaneously (the road traffic accidents number, the retention on the path, medium speed of traffic, etc.)

Essence of partial handling is that, even when there is complete information about flows and a possibility of the drivers notifications about necessary actions, these requirements are advisory in nature. In this way the control task is complicating. Presence of reliable information forecasting of drivers behavior and hard occupation of roads areas in certain time does not help to facilitate a situation of roads and leads to traffic jams. This situation is especially typical for Moscow roads.

As it is known, the methods of mathematic modelling can significantly help in the traffic control, for example research papers [3-6].

The existing approaches to modelling can be divided into two main classes: models of macro-level and models of micro-level. The models of macro-level describe a transport flow as an aggregate of all transport vehicles. Such models are frequently used for analysis of the large volume transport system (for example, highways, interregional road networks, etc.). The following are significant parameters in these models include the density of traffic. The models of micro-level are characterized by the description of separate transport vehicles and interactions between them. Basically, models of this class are used to model the motion of individual junctions and their aggregates.

\section{Control and optimization pf transport traffic using Internet- service of electronic registration}

Recently Internet-services becomes more popular, such as programs-navigators, maps, services for registration and submission of documents by citizens, signing up in policlinic on an appointment, tracking of turns at the moment in various facilities, various mobile services, etc. These services are especially convenient. Either recommendatory time or strictly determined time of visit become known for the participant of the service. Time intervals for another participants of the service are determined depending on free intervals.

We will give examples of the recommendatory and regulated time for the participant of the service.

At implementation of signing up in policlinic using the Internet-service such as emias.info or pgu.mos.ru, the participant receives the coupon for a certain date and time. The system allows not to create large turns and assumes saving of time of the participant, and also other participants of the system. Thus the time of visit is strictly regulated for the participant. Time intervals for another participants of the service are determined depending on free intervals.

Various route are offered to the participant of traffic in the presence of program-servicenavigator (the best or the worst route that depends on movement time to the specified destination). In this case internet-service provides the recommendation, but a participant can choose another route.

In their case, on optimization of road traffic depending on "random" the predictable preferences of drivers management becomes quite a challenge. The predictable preferences of movements include: seasonal movements, movements during a day (morning, day, evening, night), dependence on day of the week (weekday or day off), etc..

Despite the fact that one can call the preferences predictable, possession of this information does not always allow to optimize traffic and the traffic participant faces a situation of roads «occupation».

It is possible to refer spontaneous, unplanned trips to "stochastic" preferences.

It is offered to apply the Internet-services for creation of the regulated time interval for each participant. The model of the Internet-service can be defined as optimization problem [7]. 
The creation of such time intervals can solve the following problems:

optimization of a transport traffic for zones of roads without carrying out additional construction and expansion of the carriageway (for example, in historical center of the city),

traffic model is more predictable, participants have reliable information about traffic load not at the present moment of time, but in advance, on future time interval.

Let's review an example of the service work for a road section one need to submit an application to enter at a certain time.

For example, road users on a certain day (say, for some limited time frame) require using a section of the road A1 operated with the system of electronic passes. Users open the online service, and submit applications to enter.

They select a section of the road. Then users select a date and time they purpose to enter the zone A1 of the road. The system contains data on previous applications and "models" a traffic situation. In case the traffic does not exceed a predetermined value and the movement in the area A1 faces not difficulty the users receive an electronic ticket (electronic authorization) issued to the vehicle number entering the zone A1.

If during the requested time additional users may cause difficulties to the traffic, the system will offer an entry voucher to the A1 zone for another time slot closest to the requested one. This way the strict regulation of the traffic flow will be settled properly and the traffic will be optimized thanking to the provided management options.

Further it is necessary to consider some of problems that can appear when selecting a control style via the Internet-service.

To prevent users from violating the established movement order, the entrance of the violating vehicle to the zone without stopping are recorded and checked automatically with the database of electronic tickets (permits) regarding the time period for entering the A1 zone. If a user is not registered, he will be penalized.

In case when a user need to get to the A1 section of the road, but the system does not offer electronic tickets (for example, due to the fact there is a large number of users already registered for the next time frames, and new tickets may lead to overloading traffic), the user of the Internet-service can either select a detour for that time or purchase a ticket for a paid entry to the zone.

In this case there is a problem of calculating the spare "bunker" of paid applications for slots. It means issuing for paid tickets should not affect the deterioration of traffic conditions and road congestion due to use of paid tickets.

Today road users already know what the paid road system is $[8,9]$ and how it works as there is a good example of the Moscow-Sankt-Petersburg highway and paid roads from airports. Paid roads are also widely used abroad.

We should admit the traffic situation in Japan has been improved due to a large number of additional highways with paid concept. But there are also free options to stay aside of the main traffic.

However, the implementation of the management and optimization system is focused primarily not on the introduction of paid options for certain sections of roads only but also on traffic regulation with providing accurate information to road users about the future situation with the traffic and movement within specified road sections.

It is also necessary to calculate the time frames for every section of the road where the service will be implemented. Due to the fact that getting to a time point will be random (as drivers can not get to his destination within required time frames because of factors affecting the movement), you have to account it for a mathematical model. For different roads an allowable range may be different: 10 minutes, 15 minutes, etc.

Construct the flowchart of user registration for entering the zone. 


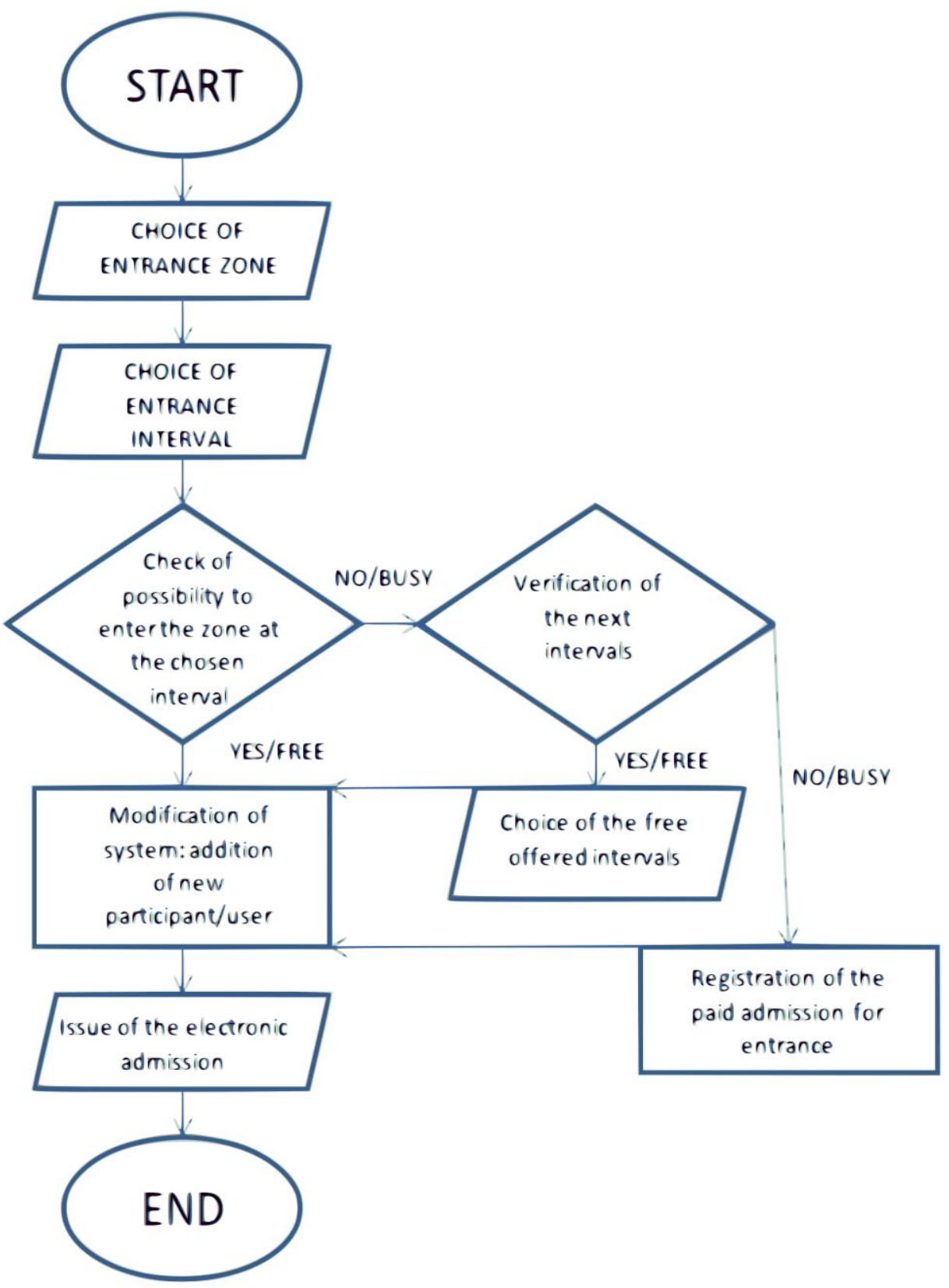

Fig. 1. Flowchart of user registration for entering the zone

The problem and principles of paid entry are now gaining popularity. In the context of managed processes (using controlled queueing models), it is possible to consider the management of the incoming flow of vehicles, taking into account the type of requests as, for example, paid and free queries. 


\section{Control on the basis of controlled queueing models}

Each queueing model includes in its structure a number of service servers and is designed to serve a flow of queries entering the system.

The aim is to make recommendations to ensure the high efficiency of the model.

For this purpose, it is necessary to establish dependencies of system functioning on its structure.

When developing mathematical models, the concept of strategy is introduced. A strategy refers to a decision-making rule, that is, model control (or model management).

For a controlled stochastic process, the problem of the optimal management strategy search is constructed. The control quality quantity must be related to a particular process path.

The management problem defines the management object. When solving a specific problem, the object can be a controlled semi-markov process. Functionality is constructed on the trajectories of controlled semi-markov processes.

Since the purpose of control is to increase the efficiency of the system operation, an objective characteristic - functionality - is selected to find strategies that determine the most productive operation of the system.

To characterize the queueing models, the symbolism proposed by Kendall using multiple bits to characterize the incoming flow (first bit), the service characteristics (second bit), the third bit defines the system structure features, the fourth bit records the queue features, the fifth bit is entered to describe the priority mass service systems.

The symbols $M, D, E, G$ denote exponential, regular, Erlangian, and arbitrary distributions, respectively. If the first bit contains one of the symbols listed above, then the incoming flow is a recurrent flow with a corresponding distribution of intervals between the times of arival of queries. Each of the characters entered above in the second bit means that the service duration distribution has the specified form. The third bit indicates the number of maintenance devices. The fourth bit indicates the number of waiting places (maximum queue length): - case of unlimited queue, - system with loss without waiting, - system with limited number of waiting places.

Given the ability to manage multiple characteristics, different options can be considered.

$M / G * / n / N$, describe the meaning of the symbols.

$M$ : The moments of arrival of the queries form the flow with parameter $\lambda$.

$G^{*}$ : The service duration distribution function varies depending on the system state, i.e. is the control effect.

$n$ : is a multi-channel system with $\mathrm{n}$ service channels.

$N$ : queued system, $\mathrm{N}$ seats.

However, it is advantageous to control the arrival-flow, which can be done using the model described below.

Note that it is very reasonable to change the characteristics of arrival flows in various models for optimization of its functioning. The control is based on the theory of controlled semi-markov processes for system optimization [10-12]. The control is carried out using a type of the next batch, the moments of batch arrivals and the quantity of queries in the batch. Various modifications to the theory of probability methods are possible for the application of control [13].

The Controlled Batch Semi-Markov Arrival Process is a generalization of the BMAPflow [14]. BMAP-flow was suggested as a good model for data-flows in telecommunication networks.

Define CBSMAP-flow [12, 15]. After holding in the state comes to an end, the Controlled Semi-Markov process jumps to the other state and the batch of queries of 
CBSMAP-flow will be generated. Note that, CBSMAP-flow is also good for modeling of data-flows and other flows.

All major characteristics for the CBSMAP-model was obtained in the previous papers [10-12].

Therefore, the following options for construction of control measures set are possible:

- control of the next batch type,

- control of the next batch type and the moment of the batch arrival,

- control of the next batch type, the moment of the batch arrival and the number of queries in the batch.

Note, that the batch type ( $i$-type) in the given problem is a type of transport devises (as cars, trucks, motorcycles). This division is important as the type of the vehicle, which arrives a transport flow, influences the total flow density. So we have queries of several different groups (batches).

The moment of the batch arrival is a "conditional" moment of vehicles arrival in a transport flow. As the real exact time of arrival is unknown, this moment is "generated" as soon as a certain number of applications/queries collects for a certain time interval. These queries (applications) form the "bunker" of the saved-up queries (applications).

The number of queries in the batch is a number of $i$-type vehicles, saved-up queries in the "bunker".

Note that CBSMAP-model can be applied for several types of admission discipline as partial admission, complete admission, complete rejection.

For describing the evolution of the system, the following algorithm is realized:

- define Markov moments,

- define the states of semi-markov process;

- define control set and control strategy;

- define semi-markov kernel and a matrix of transition probabilities for embedded Markov chain;

- construct income functional on the trajectories of controlled semi-markov process;

- define optimum strategy of control.

We define the maximal income and optimum strategy: the next batch type, the moment of the batch arrival and the number of queries in the batch.

\section{Conclusion}

The model of transport traffic optimization with use of preliminary registration is conctructed in the research. The paper demonstrated the possibility of applying the theory of controlled semi-markov queueing models to traffic flow management. There are some examples of managed models (with multiple system parameters being managed at the same time) that can be the basis for traffic management depending on the situation.

Algorithmization of model and also the main structure is presented. The example of control on the basis of CBSMAP-model is given.

The offered approach will help to reduce time spent for the road, will help traffic users to plan the route in advance.

Proposed algorithm with use of controlled models can be applied similarly to some other algorithms [16,17]. However, its application implies finding the optimal strategy in each state of the system.

Introduction of this model assumes use some state Internet-service.

At further researches in this direction many interesting tasks arises, connected with calculation of a tariff settlement for entrance to the chosen zone, with identification of optimum sites to which this model can be applied, etc. 


\section{References}

1. B. Bartosiewicz, Sz. Wiśniewski, Transport Problems 10 (3), 87-99 (2015). DOI: 10.21307/tp-2015-037

2. S.C.Calverta, H. Taaleac, M. Snelderab, S.P. Hoogendoorna, Case Studies on Transport Policy 6 (1), 81-93 (2018). https://doi.org/10.1016/j.cstp.2018.01.003

3. B. Mrówczyńska, Transport Problems 10 (3), 141-152 (2015). DOI: 10.21307/tp-2015042

4. D. García, H.A. Alonso, A.E. Molinaa, Computers \& Operations Research 112, 104768 (2019). Available at: https://doi.org/10.1016/j.cor.2019.104768

5. K. Tang, M. Boltze, H. Nakamura, Z.Tian, Road Traffic Signal Control: Principles and Global Practices (Elsevier, 2019)

6. K. Yang, M. Menendez, N. Zheng, Transportation Research Part C 105, 439-455 (2019)

7. P. M. Kumar, Usha Devi G, et.al., Computer Networks 144, 154-162 (2018)

8. A.R.Tavares, A.L. Bazzan, The Third Brazilian Workshop on Social Simulation, 99105 (2012)

9. X. Chen, Z. Zhu, L. Zhang, European transport Conference (2014)

10. E. V. Kondrashova, O.B. Zaytseva, Lecture Notes in Computer Science (including subseries Lecture Notes in Artificial Intelligence and Lecture Notes in Bioinformatics) 10684, 65-74 (2017).

11. E. V.Kondrashova, V. Kashtanov, Optimization: A Journal of Mathematical Programming and Operations Research $67 \quad$ (6), 921-941 (2018) https://doi.org/10.1080/02331934.2018.1434171

12. V. A.Kashtanov, E.V. Kondrashova, Lecture Notes in Engineering and Computer Science. Proceedings of The World Congress on Engineering 2013, 69-73 (Newswood Limited International Association of Engineers, 2013)

13. C. González, R. Clempner, B. Julio, A. Poznyak, Mathematics and Computers in Simulation (MATCOM) 166(C), 283-297 (2019) doi:10.1016/j.matcom.2019.06.002

14. D. M. Lucantoni, Communications in Statistics, Stochastic Models 7(1), 1-46 (1991)

15. E. V. Kondrashova, International Journal of Mathematical Models and Methods in Applied Sciences 10, 18-26 (2016)

16. I. Kapariasa, P. Liub, A. Tsakarestosc, et. al., Case Studies on Transport Policy (2019). https://doi.org/10.1016/j.cstp.2019.11.004

17. R. Zagidullin, Transportation Research Procedia 20, 709-716 (2017). https://doi.org/10.1016/j.trpro.2017.01.115 